\section{Case Report

\author{
Correspondence \\ Eric Chan \\ Eric.Chan@middlemore.co.nz
}

Received 10 December 2013

Accepted 24 June 2014

\title{
A rare case of early-onset neonatal sepsis
}

\author{
Eric Chan, ${ }^{1}$ Lindsay Mildenhall ${ }^{2}$ and Susan Taylor $^{1}$
${ }^{1}$ Clinical Microbiology, Laboratory, Middlemore Hospital, Auckland, New Zealand
${ }^{2}$ Consultant Neonatologist, Newborn Services, Kidz First, Middlemore Hospital, Auckland, New Zealand

\begin{abstract}
Introduction: Capnocytophaga sputigena is a capnophilic Gram-negative bacillus normally residing intra-orally in humans, and may be associated with opportunistic infections in the immunocompromised. A case of early-onset neonatal sepsis is described, which has only rarely been reported as a cause of premature birth and neonatal septicaemia.
\end{abstract}

Case presentation: This case report focuses on the rare presentation of a microbe, Capnocytophaga sputigena, causing pre-term labour, early neonatal septicaemia and maternal chorioamnionitis. To the best of our knowledge, it is the first case report of such a presentation in New Zealand. This case helps to demonstrate the contrast between the stormy clinical course in the newborn and the silent asymptomatic presentations in the mother.

Conclusion: Capnocytophaga spp. normally reside in the human oral cavity and have been associated with the pathogenesis of periodontal disease. They have also been reported in wounds in humans inflicted by animal bites, and in a wide spectrum of opportunistic infections in the immunocompromised. Only rarely have Capnocytophaga spp. been attributed as a cause associated with pre-term birth, early neonatal septicaemia or chorioamnionitis.

Keywords: antibiotics; chorioamnionitis; CPAP; fever; funiculitis; neonatal sepsis; pre-term birth; respiratory distress; septicaemia.

\section{Case report}

A 19-year-old primigravida (gravida 1, para 0) was admitted acutely to hospital because of the sudden onset of pre-term labour at 26 weeks of gestation. She was otherwise well during her pregnancy. Antenatal ultrasounds were performed at 20, 23 and 26 weeks (the last 1 day prior to delivery). Scan reports documented normal anatomy and normal intrauterine fetal growth with a normal amount of amniotic fluid. Membranes ruptured at delivery, with the liquor described as clear. There was no maternal fever and no fetal tachycardia. Antenatal steroids were administered $2 \mathrm{~h}$ prior to delivery and intravenous penicillin $(1.2 \mathrm{~g}) 90 \mathrm{~min}$ before delivery. The mother gave birth via a normal vaginal delivery to a male baby on the same day of admission.

The male baby weighed $975 \mathrm{~g}$ at birth (50\% for 26 weeks). Apgar scores were 4,4 and 7 at 1,5 and $10 \mathrm{~min}$, respectively. At delivery, the baby showed minimal respiratory effort. Heart rate dropped to less than 60 beats $\min ^{-1}$ at which point the baby was intubated and chest compressions commenced with a good response. The newborn was ventilated for $12 \mathrm{~h}$ and received continuous

Abbreviations: CPAP, continuous positive airway pressure; CRP, Creactive protein; MALDI-TOF MS, matrix-assisted laser desorption/ ionization time-of-flight mass spectrometry. positive airway pressure (CPAP) for 22 days. Serial head ultrasound scans, the first on day 5 , and magnetic resonance imaging on day 34 of life confirmed a large left-sided cerebellar haemorrhage. All through the initial stages, the baby remained afebrile.

Investigations, including blood tests and blood cultures, were performed on samples from the newborn within the first $24 \mathrm{~h}$ of life. His C-reactive protein (CRP) was noted to increase to $18 \mathrm{mg} \mathrm{l}^{-1}$ (normal range $0-5$ ), and his full blood count showed raised white blood cell counts of $2.8 \times 10^{10} \mathrm{l}^{-1}$ (normal $\left.8.0 \times 10^{9}-2.5 \times 10^{10}\right)$. The neutrophil differential count was $1.37 \times 10^{10} \mathrm{l}^{-1}$ (normal $\left.5.0 \times 10^{9}-2.1 \times 10^{10}\right)$, with a mild increase in band neutrophil forms and neutrophil toxic changes in the peripheral smears. The left shift was calculated at $13 \%$. His mobile chest X-ray revealed opacities in the left hemithorax and right upper lobe, with some volume shift to the left side. The radiological diagnosis was underventilation of the right upper lobe and the left lung. An Xray of the abdomen was normal. Initial antibiotic therapy consisted of penicillin and gentamicin. Blood cultures collected soon after delivery were flagged as positive at $24 \mathrm{~h}$ with Gram-negative bacilli. When the blood culture findings were reported to the Neonatal Unit team, penicillin was changed to cefotaxime. At $72 \mathrm{~h}$, with the organism identification, gentamicin was changed to 
amoxicillin/clavulanic acid after discussion with the regional Paediatric Infectious Diseases Service. Repeat blood cultures collected on days 37, 38 and 40 were culture negative. Despite repeated attempts, a satisfactory cerebrospinal fluid could not be obtained. Urine was not cultured.

Subcultured plates from the baby's positive blood cultures showed growth on Columbia horse blood agar (Fort Richard) and chocolate agar (Fort Richard) incubated in $5 \% \mathrm{CO}_{2}$ at $37{ }^{\circ} \mathrm{C}$. Colonies were tan coloured with spreading margins and moist centres. The colonies on blood agar plates showed no haemolysis. Microscopy with Gram staining demonstrated long, fusiform-shaped, straight to slightly curved Gram-negative bacilli.

The mother was clinically well with no symptoms or physical signs of infection and she remained afebrile. Investigations on the mother reported that there was a raised white cell count of $2.65 \times 10^{10} \mathrm{l}^{-1}$ (normal $\left.4.0 \times 10^{9}-1.5 \times 10^{10}\right)$ with an increase in differential neutrophil counts of $2.2 \times 10^{10} \mathrm{l}^{-1}$ (normal $3.4 \times 10^{9}$ $1.05 \times 10^{10}$ ). There was no significant growth from a midstream urine culture. An endocervical swab showed heavy growth of mixed genital flora including Candida albicans after culture on blood agar, chocolate agar and a split New York City/Sabouraud dextrose plate (Fort Richard). Moderate growth of tan-coloured colonies of identical appearance to the Gram-negative organism from the infant's blood was also identified. There were no Neisseria or Listeria spp. isolated.

Swabs taken from the mother's placenta over the fetal sides, between fetal membranes and on the maternal sides revealed growth of yellowish tan colonies similar to those found in the baby's blood culture and mother's endocervical swab.

The isolates were first tested with BioMérieux VITEK MS (MYLA Version 3.2.0_5. Sys. Compliance: VITEK MS 1.1.0), which was able to give a rapid identification, using MALDI-TOF MS. The MALDI-TOF MS test was positive, identifying the organism as Capnocytophaga sputigena, with a confidence value of 99.9.

The following biochemical tests were performed on the isolates obtained from the blood cultures, placental swabs and endocervical swab. They showed negative reactions with catalase, oxidase, indole and urease tests. The test for $\beta$-galactosidase was positive as it hydrolysed the colourless ortho-nitrophenyl- $\beta$-galactoside broth into ortho-nitrophenol forming a yellow colour. On testing for carbohydrate utilization, there was acid production with lactose, sucrose, maltose and glucose. All these tests were consistent with the biochemical characteristics of $C$. sputigena.

A representative isolate from the blood culture was also identified by the Institute of Environmental Science and Research as C. sputigena using $16 \mathrm{~S}$ rRNA gene sequencing, which showed $99.2 \%$ DNA sequence identity. MICs were determined using E-tests on blood agar incubated in $5 \%$
$\mathrm{CO}_{2}$. The amoxicillin MIC was $0.023 \mathrm{mg} \mathrm{l}^{-1}$ and the cefotaxime MIC was $0.016 \mathrm{mg} \mathrm{l}^{-1}$ after $48 \mathrm{~h}$. The isolate was negative for $\beta$-lactamase using a cefinase disc.

The placenta was sent for histology, and the pathology report showed funiculitis, intervillositis and chorioamnionitis in the placenta. However, despite abnormal investigation findings, the mother remained asymptomatic and afebrile, and was discharged home on the day following delivery.

The diagnosis in this case was C. sputigena infection with early-onset neonatal sepsis and maternal chorioamnionitis (with funiculitis and intervillositis), resulting in premature labour at 26 weeks of gestation. Clinically, the newborn's condition stabilized and slowly improved over the following 13 weeks. His CRP and white cell counts gradually returned to normal. His chest X-ray cleared. He continued to have no fever. The antibiotics were stopped after 4 weeks on resolution of the C. sputigena septicaemia.

However, approximately 2 weeks after cessation of antibiotics, the baby developed a fever $\left(38.9{ }^{\circ} \mathrm{C}\right)$ for 2 days. His CRP then increased to $7 \mathrm{mg} \mathrm{l}^{-1}$, but his white cell and differential counts were normal. A septic screen was carried out, and antibiotics were started empirically with amoxicillin and amikacin due to concern regarding recurrent sepsis and meningitis. The results of investigations of the septic screen, including cerebrospinal fluid examination and PCR for respiratory viruses including influenza $A$ virus, H1N1 2009 influenza A virus, influenza B virus, respiratory syncytial virus, parainfluenza viruses 1, 2 and 3, adenovirus types 1-8, human metapneumovirus (using nasopharyngeal aspirate tested with an AusDiagnostics multiplexed-tandem PCR Respiratory Pathogen Panel) were all negative. His fever subsided after 2 days, and the antibiotics were accordingly stopped.

After 13 weeks, the baby was discharged home, clinically stable, and will be followed up in the Outpatient Clinic. Although his left cerebellar bleed remained, the associated abnormality in left Sylvian fissure had resolved. The CPAP was no longer needed. He gained weight over the last 2 months and, despite his initial very low birth weight, he weighed 2920 g. Nutritionally, the baby did well on oral feeds and was making a slow but steady progress towards recovery.

\section{Discussion}

Capnocytophaga is a genus of facultative anaerobic, fastidious, fusiform, non-spore-forming Gram-negative bacilli that normally reside in the oral cavity and are rarely found in the female genital tract. There are nine Capnocytophaga spp. (Versalovic et al., 2011), comprising C. gingivalis, C. ochracea, C. haemolytica, C. sputigena, C. granulosa, C. leadbetteri, genospecies AHN8471, C. canimorsus and C. cynodegmi. The first seven are normally found in the human 
oral cavity, whilst the last two are found as dog or cat oral flora.

In this case, the clinical manifestations were peculiar with a prominent absence of symptoms or signs in the mother's apparently silent subclinical chorioamnionitis in contrast to the initial stormy clinical course of early neonatal sepsis in her newborn child.

A literature search for pre-term births in association with Capnocytophaga infections in early gestation in pregnancy revealed 22 such cases (Douvier et al., 1999; Howlett et al., 2007; Iralu et al., 1993; Lopez et al., 2010; Mekouar et al., 2012; Mikamo et al., 1996; Vandemeulebrouck et al., 2008) and, up to 2012, there were only three cases of proven neonatal septicaemia with positive cultures of Capnocytophaga sputigena isolated from blood.

Our case constitutes the fourth case of proven early neonatal septicaemia, based on the evidence of positive cultures of C. sputigena from neonatal blood samples and placental swab samples. This, coupled with the manifestations of neonatal respiratory distress, the abnormal pulmonary chest X-ray findings and the presence of leukocytosis with an increase in white cell counts and neutrophil differential count at birth, all lend support to the diagnosis of early neonatal sepsis.

In culture-negative cases of women with chorioamnionitis and pre-term labour, molecular methods such as $16 \mathrm{~S}$ rRNA gene PCR and sequencing can be used to identify bacteria such as Capnocytophaga spp. However, as this molecular technique is not routinely available, cases of preterm labour due to Capnocytophaga infection may be missed, especially when the presentation of chorioamnionitis is clinically silent and asymptomatic, or when isolates are not recovered from cultures.

Normally, Capnocytophaga spp. can be isolated from the oral cavity associated with the development of periodontitis (Hansen et al., 1996; Mikamo et al., 1996) in immunocompetent persons. They have also been described as responsible for a wide variety of infections (Bonatti et al., 2003; Howlett et al., 2007; Lopez et al., 2010; Mekouar et al., 2012; Vandemeulebrouck et al., 2008; Versalovic et al., 2011), such as septicaemia, meningitis, endocarditis, or wound and chest infections, especially in those with immunodeficiency.

Capnocytophaga spp. are thought to reach the uterus either by ascending through the cervix after orogenital contact from a partner with periodontal disease (Hansen et al., 1996; Mikamo et al., 1996) or by haematogenous spread to the placenta, resulting in sepsis in the fetus.

Usually, Group B streptococci or Escherichia coli are responsible for chorioamnionitis and infection of the amniotic fluid through ascending infection after the membranes have ruptured, whereas Bacteroides spp., genital mycoplasmas or Ureaplasma spp. are responsible for chorioamnionitis in pre-term labour with intact membranes (Goldenberg et al., 2000; Tita \& Andrews, 2010). Capnocytophaga spp., such as C. sputigena, are found only rarely in cases of pre-term labour associated with chorioamnionitis and neonatal sepsis.

Virulence factors of Capnocytophaga spp. (Huck et al., 2011; Sbordone \& Bortolaia, 2003; Spratt et al., 1995) have been detected from isolates of patients with periodontitis or oral lesions. These virulence factors, consisting of enzymes produced by Capnocytophaga spp. (e.g. aminopeptidases and proteases), can cause destruction and damage to periodontal tissues, with production of inflammatory molecules. Hypothetically, the production of inflammatory cytokines may result in their entry into the blood stream, leading to the development of chorioamnionitis.

Capnocytophaga spp. are usually susceptible (Mekouar et al., 2012; Versalovic et al., 2011) to macrolides (e.g. erythromycin and azithromycin), lincosamides (e.g. clindamycin), carbapenems, third-generation cephalosporins, tetracyclines and fluoroquinolones. However, it has been noted that $\beta$-lactamase can be produced by some Capnocytophaga spp., which may then be resistant to penicillins or cephalosporins (including third-generation cephalosporins), and this resistance to $\beta$-lactams has been reported in patients with leukaemia or neutropenia, associated with $\beta$-lactamase-producing Capnocytophaga spp. (Gomez-Garces et al., 1994; Sébastien et al., 1999). Capnocytophaga spp. are usually resistant to aminoglycosides (e.g. gentamicin, amikacin and tobramycin) and colistin.

\section{Conclusion}

Capnocytophaga spp. normally reside in the human oral cavity and have been associated with the pathogenesis of periodontal disease. They have also been reported in the wounds in humans inflicted by animal bites, and in a wide spectrum of opportunistic infections in the immunocompromised. Only very rarely are Capnocytophaga spp. attributed as a cause associated with pre-term birth, early neonatal septicaemia or chorioamnionitis.

\section{Acknowledgements}

Many thanks are expressed to the Institute of Environmental Science and Research, especially Kristin Dyet and Heather Davies, for performing the $16 \mathrm{~S}$ rRNA gene sequencing on our culture isolate, and to our Middlemore Hospital Laboratory team for their technical support. The authors do not have any association that might constitute a conflict of interest.

\section{References}

Bonatti, H., Rossboth, D. W., Nachbaur, D. W., Fille, D. M., Aspöck, C., Hend, I., Hourmont, K., White, L., Malnick, H. \& Allerberger, F. J. (2003). A series of infections due to Capnocytophaga spp in 
immunosuppressed and immunocompetent patients. Clin Microbiol Infect 9, 380-7.

Douvier, S., Nenwinth, C., Fillipuzzi, L. \& Kisterman, J. P. (1999). Chorioamnionitis with intact membranes caused by Capnocytophaga sputigena. Eur J Obstet Gynecol Reprod Biol 83, 109-112.

Goldenberg, R. L., Hauth, J. C. \& Andrews, W. W. (2000). Intrauterine infection and preterm delivery. N Engl J Med 342, 1500-1507.

Gomez-Garces, J. L., Alos, J. I., Sanchez, J. \& Cogollos, R. (1994). Bacteremia by multidrug-resistant Capnocytophaga sputigena. J Clin Microbiol 32, 1067-1069.

Hansen, L. M., Dorsey, T. A., Batzer, F. A. \& Donnerfeld, A. E. (1996). Capnocytophaga chorioamnionitis after oral sex. Obstet Gynecol 88, 731.

Howlett, A. A., Mailman, T. L. \& Ganapathy, V. (2007). Early cystic lung disease in a premature neonate with perinatally acquired Capnocytophaga. J Perinatol 27, 68-70.

Huck, O., Tenenbaum, H. \& Davideau, J. L. (2011). Relationship between periodontal diseases and preterm birth: recent epidemiological and biological data. J Pregnancy 2011, 164654.

Iralu, J. V., Roberts, D. \& Kazanjian, P. H. (1993). Chorioamnionitis caused by Capnocytophaga: case report and review. Clin Infect Dis 17, 457-461.

Lopez, E., Raymond, J., Patkai, J., El Ayoubi, M., Schmitz, T., Moriette, G. \& Jarreau, P. H. (2010). Capnocytophaga species and preterm birth: case series and review of the literature. Clin Microbiol Infect 16, 1539-1543.
Mekouar, H., Voortman, G., Bernard, P., Hutchings, G., Boeras, A. \& Rodriguez-Villalobos, H. (2012). Capnocytophaga species and perinatal infections: case report and review of the literature. Acta Clin Belg 67, 42-45.

Mikamo, H., Kawazoe, K., Sato, Y., Izumi, K. \& Tamaya, T. (1996). Intra-amniotic infection caused by Capnocytophaga species. Infect Dis Obstet Gynecol 4, 301-302.

Sbordone, L. \& Bortolaia, C. (2003). Oral microbial biofilms and plaque-related diseases: microbial communities and their role in the shift from oral health to disease. Clin Oral Invest 7, 181-188.

Sébastien, M., Leblanc, T., Rousselot, P., Legrand, P., Arlet, G. \& Cordonnier, C. (1999). Bacteremia due to Capnocytophaga species in patients with neutropenia: high frequency of $\beta$-lactamase-producing strains. Clin Infect Dis 28, 1172-1174.

Spratt, D. A., Greenman, J. \& Schaffer, A. G. (1995). Capnocytophaga gingivalis aminopeptidase : a potential virulence factor. Microbiology 141, 3087-3093.

Tita, A. T. N. \& Andrews, W. A. (2010). Diagnosis and management of clinical chorioamnionitis. Clin Perinatol 37, 339-354.

Vandemeulebrouck, B., Rasigade, J. P., Sobas, C., Vialet, A. \& Roubille, M. (2008). [Septicemia with Capnocytophaga sputigena in a newborn child]. Ann Biol Clin 66, 215-219, (in French).

Versalovic, J., Carroll, K. C., Funke, G., Jorgensen, J. H., Landry, M. L. \& Warnock, D. W. (2011). Capnocytophaga. In Manual of Clinical Microbiology, 10th edn, pp., 574-578, Washington, DC: ASM Press. 